\title{
Effectiveness of non-invasive ventilation in acute respiratory failure of obese patients: a brief narrative review
}

\author{
Maurizio Alessandro Cavalleri, Elena Barbagelata, Marco Scudeletti, Antonello Nicolini \\ Department of Internal Medicine, General Hospital, Sestri Levante (GE), Italy
}

\begin{abstract}
Non-invasive ventilation (NIV) has been used successfully for the management of acute respiratory failure (ARF) more often in the last two decades compared to prior decades; nevertheless, NIV can have failure rates ranging from $5 \%$ to $50 \%$. However, there are particular groups of patients that are more likely to benefit from NIV. One of these groups is patients with hypoventilation syndrome (OHS). The aim of this review is to seek evaluation of the effectiveness of NIV in acute setting. Only a few studies have investigated NIV success or failure in OHS patients. More than $30 \%$ of them were diagnosed when hospitalized for ARF. NIV rarely failed in reversing ARF. OHS patients who exhibited early NIV failure had a high severity score and a low $\mathrm{HCO}_{3}$ level at admission; more than half of hypercapnic patients with decompensated OHS exhibited a delayed but successful response to NIV. Patients with decompensation of OHS have a better prognosis and response to NIV than other hypercapnic patients. They require more aggressive NIV settings, a longer time to reduce $\mathrm{PaCO}_{2}$ levels, and more frequently a delayed but successful response to NIV.
\end{abstract}

\section{Introduction}

Obesity hypoventilation syndrome (OHS) is defined as a combination of obesity (body mass index $>30 \mathrm{~kg} / \mathrm{m}^{2}$ ), daytime hypercapnia, $\mathrm{PaCO}_{2}>45 \mathrm{mmHg}$, and disordered breathing during sleep. ${ }^{1-3}$ OHS is a chronic disease associated with respiratory and cardiometabolic impairments leading to a decrease in ability to perform normal daily activities including social interactions along with a higher risk of hospitalization and death. ${ }^{2,3}$ The disease is largely under recognized

Correspondence: Maurizio Cavalleri, Department of Internal Medicine, General Hospital, via Terzi 43, 16039 Sestri Levante (GE), Italy.

Tel.: +39.0185.329904.

E-mail: mcavalleri.doc@hotmail.it

Key words: Obesity-hypoventilation syndrome; critically ill obese patients; non-invasive ventilation; late failure.

Conflict of interest: the authors declare no potential conflict of interest.

Received for publication: 19 August 2017.

Revision received: 2 November 2017.

Accepted for publication: 5 December 2017.

This work is licensed under a Creative Commons Attribution NonCommercial 4.0 License (CC BY-NC 4.0).

CCopyright M.A. Cavalleri et al., 2018

Licensee PAGEPress, Italy

Italian Journal of Medicine 2018; 12:5-9

doi:10.4081/itjm.2018.916 and often discovered when acute respiratory failure occurs. ${ }^{2,3}$ Non-invasive ventilation (NIV) is being increasingly used for the management of acute and chronic respiratory failure. Over the past two decades, increasing evidence in literature has confirmed the main role of NIV in first line therapy for certain forms of acute respiratory failure (ARF), ${ }^{4}$ particularly in ARF due to acute exacerbations of chronic obstructive pulmonary disease (COPD), and cardiogenic pulmonary. ${ }^{5-7}$ Its usefulness has also been confirmed in patients who are immuno-compromised from hematological diseases. ${ }^{7}$ Over the time NIV was used in more severe patients and several clinical situations with proven efficacy (e.g., restrictive lung diseases, obesity hypoventilation syndrome and weaning from invasive mechanical ventilation). ${ }^{7}$ Since NIV can have failure rates ranging from $5 \%$ to $50 \%$ the delayed recognition of NIV failure may cause intubation delay, increasing morbidity and mortality. ${ }^{7}$ This review seeks to demonstrate how to determine which obese patients are good candidates, which patients are poor candidates, and how to gauge the success (or failure) of NIV rapidly (Figure 1).

\section{Data source}

We searched the following electronic databases from their inception to January 2001 to December 2016: MEDLINE, EMBASE, CINHAIL, CENTRAL (Cochrane Central register of Controlled Trials), DARE (Database of Abstracts of Reviews of Effectiveness), the Cochrane Database of Systematic Reviews, ACP Journal Club database. 
No language restrictions were applied: nonEnglish publications were professionally translated into English.

\section{Study selection}

We identified studies that included adults admitted to acute care hospitals with acute or acute on chronic

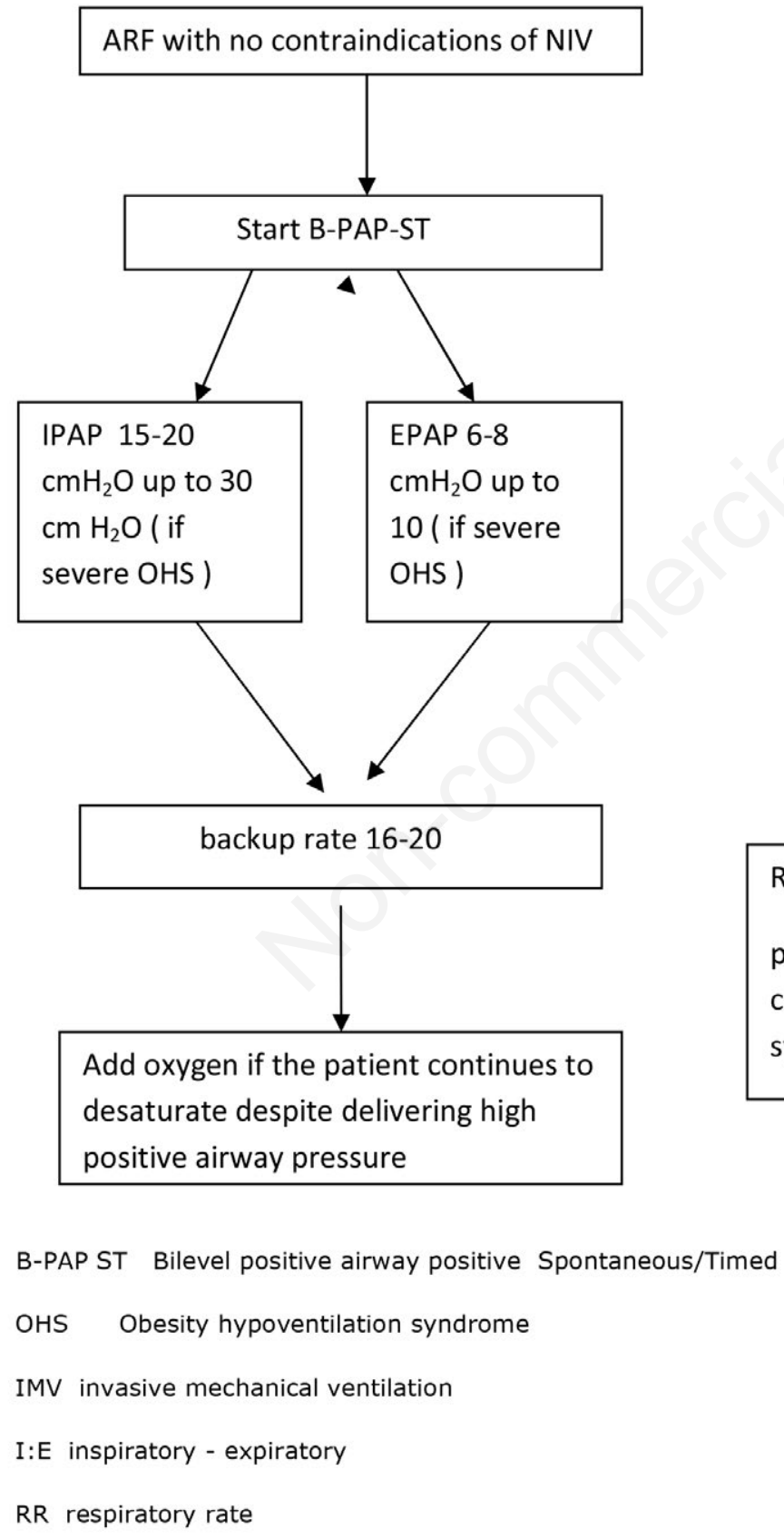

respiratory failure who needed NIV. We excluded studies of invasive mechanical ventilation. We included both randomized controlled trials and prospective studies, and excluded case reports, letters, and editorials. We have found 122 potentially eligible references; of these, 108 abstracts were excluded because they did not fulfill inclusion criteria. We read the remaining 14 papers in full text and we included all of them in the review.

\section{Oxygenation aim 88-92\%.If high \\ flow oxygen need : consider IMV}

Red flags

$\mathrm{pH}<7.25$ on optimal NIV, RR $>25$, new onset confusion despite optimal patient-ventilator synchronization, mask fit : consider IMV

Figure 1. Non-invasive ventilation (NIV) setup for obese patients with acute respiratory failure (ARF). 


\section{Results}

Patients who are likely to benefit from NIV need to be identified early. ${ }^{7,8}$ Failure to do so often results in increased morbidity and mortality as well as inappropriate use of limited resources. ${ }^{9}$ Before starting NIV, it is crucial to identify good candidates. ${ }^{7}$ In fact NIV efficacy depends on careful patient selection; the best way to do this is to look for predictors of failure immediately. ${ }^{9}$ The absence of the essential qualifications of alertness and an ability to follow instructions exclude many patients. The risk of NIV failure determines the intensity of monitoring needed. ${ }^{9}$ Moreover, knowing the factors affecting the NIV success may help to decide also the duration of NIV trial. One approach to determine the need for monitoring is to assess the patient's risk of NIV failure. ${ }^{10}$ Some of these are simple bedside assessments, such as ease of arousability, agitation, cough integrity, and respiratory rate. Other methods require simple laboratory tests, such as determination of arterial blood gases. Other methods require proven evaluation protocols [e.g., acute physiology and chronic health evaluation (APACHE) II or simplified acute physiology score (SAPS) II]. When a quick decision is required, reliance on simple bedside observations and rapidly obtained laboratory values such as $\mathrm{pH}$ is the best choice. ${ }^{10,11}$ Recognition that NIV is failing is an important, but often overlooked, part of clinical management. ${ }^{9-12}$ A patient with multiple risk factors for NIV failure should be in a closely monitored setting such as an Intensive Care Unit (ICU) or a step-down respiratory unit. Three critical periods for detecting NIV failure have been defined: i) immediate failure (within minutes to $<1$ h); ii) early failure ( 1 to $48 \mathrm{~h}$ ); and iii) late failure (after $48 \mathrm{~h}$ ). ${ }^{9-11}$

\section{Immediate non-invasive ventilation failure}

Immediate NIV failure refers to failure within sixty minutes. ${ }^{11}$ Predictors of failure in this period have never been systematically analyzed. A weak cough reflex leading to inefficient clearance of excessive secretions from airways is a common cause of immediate NIV failure. The inability to spontaneously remove secretions is considered a contraindication to NIV, e.g., patients with impaired consciousness and depressed cough reflex. Hypercapnic encephalopathy is often considered a cause of immediate NIV failure because of poor compliance due to confusion and/or agitation. It is a relative contraindication because of the increased risk of aspiration. ${ }^{9,13-15}$ The risk of aspiration has been shown to be minimized by the rapid improvement of neurological status. Patient tolerance has been shown to be critical for NIV success, especially in the first few minutes while the patient adapts to this new mode of breathing. ${ }^{15}$

\section{Early non-invasive ventilation failure}

Nearly $65 \%$ of NIV failures occur within $1-48 \mathrm{~h}$ of NIV use. This time interval has received more attention in assessments of NIV failure than any other. Several investigators have tried to assess the best predictors of NIV failure. Confalonieri et al. found that subjects likely to fail NIV had more severe respiratory acidosis, a lower level of consciousness, were older, more hypoxemic, and had a higher respiratory rate on presentation. ${ }^{16}$ Clinical signs that are only equivocal on presentation become more definitively predictive of failure if they persist after 2 $\mathrm{h}$ of NIV. It is important to assess clinical trajectory after 1-2 h of initiation of NIV to identify response. However, even on presentation, subjects who have a $\mathrm{pH}<7.25$, an APACHE II score $>29$, and a Glasgow coma score $<11$ have failure rates ranging from $64 \%$ to $82 \%$ are at risk for early failure. Patients with excessive respiratory secretions or without improvement after 60 minutes of NIV have high risk of failure. ${ }^{10,15,16}$

\section{Late non-invasive ventilation failure}

Late NIV failure (after $48 \mathrm{~h}$ ) is an event occurring after an initial good response to NIV. It is more likely in subjects who, at the time of admission, showed functional limitations evaluated using a score correlated to activities of daily living, a higher number of comorbidities, a lower $\mathrm{pH}$ at baseline and the underlying cause of ARF, e.g., pneumonia., ${ }^{70,14,15}$

\section{Obesity hypoventilation syndrome and acute respiratory failure}

More than $30 \%$ of patients are diagnosed when hospitalized for acute on chronic respiratory failure. 5-7,13,17-21 Patients with OHS often respond well to NIV. The pathophysiology of OHS results from complex interactions between various sleep breathing disorders (obstructive sleep apnea, REM sleep hypoventilation), increased work of breathing due to a decreased thoraco-abdominal compliance, and altered ventilatory drive. ${ }^{2,3,21}$ Moreover, obesity increases the stiffness of the total respiratory system with consequent development of low lung volume. ${ }^{2,3}$

Breathing at low volumes increases airway resistance with expiratory flow limitation and gas trapping due to early airway closure and subsequent generation of intrinsic positive end-expiratory pressure and ventilation mismatching especially in supine position and during sleep., ${ }^{2,3}$ These mechanisms can be successfully corrected by NIV. Patients with OHS can be treated in an acute care 
setting with better results than other diseases such as COPD. NIV reduces the respiratory load, increases minute volume for a given breathing effort and provides ventilation during central apneic events. ${ }^{19,22,23}$ The decreased responsiveness in hypoxic and hypercapnic ventilatory drive that characterizes this last category may explain why they need more time than expected to correct respiratory acidosis compared to other patients (e.g., COPD patients). ${ }^{7,13}$ No randomized controlled trials comparing different forms of NIV for acute decompensated respiratory failure have been performed. ${ }^{2}$ The number of patients in the studies evaluated in our review ranked from $20^{5}$ to $173 .{ }^{19}$ The lower NIV failure was $6 \%$, reported by Carrillo et al. ${ }^{19}$ the higher was $41 \%,{ }^{4}$ whereas the higher mortality rate was observed in the study of Lemyze et al. ${ }^{10}$ and the lower in that of Gursel $(0 \%){ }^{4}$

No ventilatory mode has been shown to lead to better outcome than others. ${ }^{23-26}$ Several modes have been implemented: continuous positive airway pressure (CPAP), bilevel positive airway pressure (Bi$\mathrm{PAP})$, pressure support ventilation, assisted pressure control ventilation (APCV), Bi-PAP-spontaneoustimed (ST) with volume assured pressure support. $3,13,19,20,22,25-27$ More recently the so called highintensity ventilation (APCV with high rate back-up) has demonstrated encouraging results in terms of $\mathrm{PaCO}_{2}$ reduction and mortality. ${ }^{28}$ The setting of ventilator is very important because the delay in the reduction of $\mathrm{PaCO}_{2}$ may be due to:

- Inadequate level of inspiratory (IPAP) or expiratory (EPAP) pressure: in obese patients IPAP should range from 12 to $30 \mathrm{H}_{2} \mathrm{O}$ or greater and EPAP from 8 to $12 \mathrm{~cm} \mathrm{H}_{2} \mathrm{O} .{ }^{7}$ High thoracic impedance in OHS patients requires higher IPAP and EPAP levels in order to prevent the collapse of the upper airway. EPAP should be increased gradually until snoring, witnessed apneas and dips in oxygen saturation disappear. Pressure support ( $\triangle$ IPAP-EPAP) should be increased to ensure adequate tidal volume and optimal ventilation. ${ }^{3}$ Oxygen therapy must be added if the patient continues to desaturates despite high positive airway pressure. ${ }^{3,7,25} \mathrm{~A}$ back-up rate is mandatory because central apneas commonly occur. $^{3}$

- Inadequate duration of NIV: the obese patients as previously explained require longer NIV therapy than non-obese patients especially during the night; their apneas or hypoventilation worsen during hours of sleep. These findings suggest that NIV settings need to be monitored more aggressively in OHS than in other syndromes when acute illness precipitates respiratory decompensation (especially pneumonia). ${ }^{13,27}$

\section{Non-invasive ventilation setting}

Most of the studies which have evaluated NIV in OHS patients were performed in a ICU setting $4,5,8,10,12,19,24,26,27$ and the others in an intermediate respiratory care unit. $9,17,20,22,25$ This is due to the strict and more aggressive monitoring needed for these patients. ${ }^{3,10,25,26}$ The most used ventilators have been NIV platform ventilators or ICU ventilators. In a study, a home ventilator was also used in Bi-PAP-ST or CPAP mode..$^{24}$ NIV settings need to be monitored more aggressively in OHS (when acute illness precipitates respiratory decompensation) than in other diseases. ${ }^{3,10,20,25}$ During NIV therapy, observation and monitoring of the level of consciousness, vital signs, respiratory pattern, oxygen saturation and arterial blood gases are crucial. ${ }^{20,23,25,26}$ NIV modality and ventilator setting during ARF episode should be adjusted based titration with polysomnography. $3,25,26$

\section{Predictors of non-invasive ventilation failure}

NIV failure rates from 2 to $60 \%$. $^{3-5,8,10,17,19,24-27}$ Lemyze et al. ${ }^{10}$ and Contou et al. ${ }^{27}$ showed higher NIV failure (60\% and $38 \%$ respectively) and mortality rate (30\% and $14 \%$ respectively) because they enrolled more severe patients. In the other studies NIV failure ranged approximately from $2 \%$ to $41 \%$. Mortality rates were assessed only in $30 \%$ of the other studies and ranged from 0 to $6 \%$. Only one study addressed predictors of NIV failure in OHS patients. ${ }^{10}$ This study has shown multiorgan failure is the main factor associated with early NIV failure and hospital mortality. OHS patients who exhibited early NIV failure had a high severity score at admission. Lower $\mathrm{pH}$ and higher $\mathrm{PaCO}_{2}$ did not correlate with higher NIV failure and mortality. $4,8,10,19$

\section{Limitations}

This study has some limitations: it is not a systematic review, and the scarce number of studies do not allow us to provide definitive conclusions. Second, based on previous considerations, the proposed ventilation strategies are based on expertise of a single center. The use of the type of interface (total face mask or helmet) as well as side-effects or managing problems (leakage, gastric inflation) have not been addressed in this review because these were not reported in the studies we have evaluated: we are aware of their importance in the success or failure of NIV trial.

\section{Conclusions}

In conclusion, although few studies are available concerning morbidly obese patients with decompen- 
sation of OHS, they suggest that these patients require more aggressive NIV settings, a longer time to reduce $\mathrm{PaCO}_{2}$ levels below $50 \mathrm{mmHg}$, and they exhibit more frequently a delayed but successful response to NIV. OHS patients are frequently misdiagnosed as having COPD or asthma when admitted for an episode of ARF. An early diagnosis can avoid several admissions to ICU.

\section{References}

1. Selim BJ, Ramar K, Surani S. Obesity in the intensive care unit: risks and complications Hosp Pract 2016;44:146-56.

2. Piper A. Obesity hypoventilation syndrome. Chest 2016;149:856-68.

3. Sequeira TCA, Ba Hammam A, Esquinas AM. Noninvasive ventilation in the critically ill patient with obesity hypoventilation syndrome: a review. J Intensive Care Med 2016 [Epub ahead of print].

4. Gursel G, Aydodgu M, Tasyurek S, et al. Factors associated with non invasive ventilation response in the first day of therapy in patients with hypercapnic respiratory failure. Ann Thorac Med 2012;7:92-7.

5. Ciledag A, Kaya A, Akdogan BB, et al. Early use of noninvasive mechanical ventilation in patients with acute hypercapnic respiratory failure in respiratory ward: a prospective study. Arch Bronconeumol 2010;46:538-42.

6. Pacilli AMG, Valentini I, Carbonara P, et al. Determinants of noninvasive ventilation outcomes during an episode of acute hypercapnic respiratory failure in chronic obstructive pulmonary disease: the effects of comorbidities and causes of respiratory failure. BioMed Res Intern 2014;2014:976783.

7. Davidson AC, Banham S, Elliott M, et al. BTS/ICS guidelines for the ventilatory management of acute hypercapnic failure in adults. Thorax 2016;71:ii1-35.

8. Kaya A, Ciledag A, Cayli I, et al. Associated factors with non-invasive ventilation failure in acute hypercapnic respiratory failure. Tuberk Tor Der 2010;58:128-34.

9. Moretti M, Cilione C, Marchioni A, et al. Incidence and causes of non-invasive mechanical ventilation failure after initial success. Thorax 2000;55:819-25.

10. Lemyze M, Taufour P, Duhamel A, et al. Determinants of noninvasive ventilation success or failure in morbidly obese patients in acute respiratory failure. Plos One 2014;9:e97563.

11. Ozyilman E, Ugurlu AO, Nava S. Timing of non invasive ventilation failure: causes, risk factors, and potential remedies. BMC Pulm Med 2014;14:19.

12. Aboussouan LS, Ricaurte B. Noninvasive positive pressure ventilation: increasing use in acute care. Clev Clin J Med 2010;77:307-16.

13. Bello G, De Pascale G, Antonelli M. Non invasive ventilation:practical advise. Curr Opin Crit Care 2013;19:1-8.

14. Berg KM, Clardy P, Donnino MW. Noninvasive ventilation for acute respiratory failure: a review of the literature and current guidelines. Intern Emerg Med 2012;7:539-45.

15. Carratu' P, Bonfitto P, Dragonieri S, et al. Early and late failure of noninvasive ventilation in chronic obstructive pulmonary disease with acute exacerbation. Eur J Clin Invest 2005;35:404-9.

16. Confalonieri M, Garuti G, Cattaruzza MS, et al. A chart of failure risk for noninvasive ventilation in patients with COPD exacerbation. Eur Respir J 2005;25:348-55.

17. Masa JF, Utrabo I, Gomez de Terreros J, et al. Noninvasive ventilation for severely acidotic patients in respiratory intermediate care units. BMC Pulm Med 2016;16:97.

18. Piroddi IMG, Karamichali S, Barlascini C, et al. Obesity and breathing related sleep disorders: concise clinical review. SMJ Pulm Med 2015;1:1002.

19. Carrillo A, Ferrer M, Gonzalez-Diaz G, et al. Noninvasive ventilation in acute hypercapnic respiratory failure caused by obesity hypoventilation syndrome and chronic obstructive pulmonary disease. Am J Resp Crit Care Med 2012;186:1279-85.

20. Hodgson LE, Murphy PB, Hart N. Respiratory management of the obese patient undergoing surgery. $\mathrm{J}$ Thor Dis 2015;7:943-52.

21. Esquinas AM, Bahamman AS. The emergent malignant obesity hypoventilation syndrome: a new critical care syndrome. J Intens Care Med 2012;28:198-9.

22 . Hess DR. Non invasive ventilation for acute respiratory failure. Respir Care 2013;58:950-69.

23. Nava S. Behind a mask: tricks, pitfalls and prejudices for noninvasive ventilation. Respir Care 2013;58:1367-76.

24. Gursel G, Aydogdu M, Gulbas G, et al. The influence of severe obesity on non-invasive ventilation (NIV) strategies and responses in patients with acute hypercapnic respiratory failure attacks in the ICU. Min Anestesiol 2011;77:17-25.

25. Ba Hammamm AS, Al-Jawder EA. Managing acute respiratory decompensation in the morbidly obese. Respirol 2012;17:759-71.

26. Marek PE. The malignant obesity hypoventilation syndrome (MOHS). Obes Rev 2012;13:902-9.

27. Contou D, Frugnoli C, Cordoba-Izquierdo A, et al. Noninvasive ventilation for acute hypercapnic respiratory failure: intubation rate in an experienced unit. Respir Care 2013;58:2045-52.

28. Blankenburg T, Benthim C, Pohl S, et al. Survival of hypercapnic patients with COPD and obesity hypoventilation syndrome treated with high intensity ventilation in the daily routine care. Open Respir Med J 2017;11:31-40. 